\title{
COMBINING 3D VOLUME AND MESH MODELS FOR REPRESENTING COMPLICATED HERITAGE BUILDINGS
}

\author{
F. Tsai ${ }^{1,2}$, H. Chang ${ }^{2}$ and Y.-W. Lin ${ }^{2}$ \\ ${ }^{1}$ Center for Space and Remote Sensing Research \\ ${ }^{2}$ Department of Civil Engineering \\ National Central University \\ 300 Zhongda Road, Zhongli Taoyuan, 320, Taiwan \\ ftsai@csrsr.ncu.edu.tw; 1984chang@gmail.com
}

KEY WORDS: Building Modeling, 3D Model, Mesh Model, Point Clouds, Level of Detail

\begin{abstract}
:
This study developed a simple but effective strategy to combine 3D volume and mesh models for representing complicated heritage buildings and structures. The idea is to seamlessly integrate 3D parametric or polyhedral models and mesh-based digital surfaces to generate a hybrid 3D model that can take advantages of both modeling methods. The proposed hybrid model generation framework is separated into three phases. Firstly, after acquiring or generating 3D point clouds of the target, these 3D points are partitioned into different groups. Secondly, a parametric or polyhedral model of each group is generated based on plane and surface fitting algorithms to represent the basic structure of that region. A "bare-bones" model of the target can subsequently be constructed by connecting all $3 \mathrm{D}$ volume element models. In the third phase, the constructed bare-bones model is used as a mask to remove points enclosed by the bare-bones model from the original point clouds. The remaining points are then connected to form 3D surface mesh patches. The boundary points of each surface patch are identified and these boundary points are projected onto the surfaces of the bare-bones model. Finally, new meshes are created to connect the projected points and original mesh boundaries to integrate the mesh surfaces with the $3 \mathrm{D}$ volume model. The proposed method was applied to an open-source point cloud data set and point clouds of a local historical structure. Preliminary results indicated that the reconstructed hybrid models using the proposed method can retain both fundamental $3 \mathrm{D}$ volume characteristics and accurate geometric appearance with fine details. The reconstructed hybrid models can also be used to represent targets in different levels of detail according to user and system requirements in different applications.
\end{abstract}

\section{INTRODUCTION}

Detailed and highly accurate three-dimensional (3D) modeling, reconstruction, and visualization of historical monuments and cultural buildings or objects is one of the most commonly employed approaches in digital documentation and preservation of cultural heritage and their applications. Although digital 3D reconstruction or modeling of cultural heritage targets has been carried out for decades and a variety of related instruments, algorithms and softwares have been proposed and developed, it still remains as one of the most challenging topics in digital documentation of cultural heritage in terms of research and practice (Koller et al., 2009; Münster et al., 2015). In recent years, 3D point clouds have become one of the most popular data for describing complicated heritage buildings or structures in great details. These point clouds can be acquired directly with different sensors and instruments such as laser scanning (or LiDAR, Light Detection and Ranging) equipments, or generated from images captured using digital optical cameras and range (depth) cameras or sensors with close-range photogrammetry algorithms.

Digital documentation of cultural heritage usually requires highdensity point cloud data. In this regard, terrestrial laser scanning (TLS) has been a popular instrument of choice for data acquisition. However, LiDAR-based point cloud data acquisition systems are usually (relatively) expensive and time consuming. Recently, image-based point cloud generation with photogrammetric matching algorithms have emerged as a popular alternative for

\footnotetext{
* \{corresponding author $\}$
}

obtaining high quality 3D point cloud data (e.g., Alshawabkeh et al., 2010; Dellepiane et al., 2013; Koutsoudis et al., 2014) or used in conjunction with laser scanned data sets (Jamhawi et al., 2016).

For most applications, the point clouds are usually further processed to produce 3D models of the targets. A fast and economic modeling approach is connecting all points to form a network (mesh) describing the surface geometry, shape, and other property of the target surface. Another approach is to create a parametric, polyhedral, prismatic model deduced from the point clouds.

In order to take advantages of both approaches, this research developed a simple coarse-to-fine modeling framework to combine $3 \mathrm{D}$ volume and mesh models for representing complicated heritage buildings and structures. The developed model generation framework first reconstruct bare-bones models from 3D point clouds of complicated cultural heritage buildings. The bare-bones model consists of parametric or polyhedral elements of the target, which are used as the skeleton to describe the basic structure of a complicated building. Then mesh-based surface model patches are generated and integrated with the bare-bones model to describe complicated surface decorations or other non-skeleton objects. Using this strategy, the reconstructed hybrid building model will maintain fundamental structural characteristics as well as fine details of complicated surface objects. In addition, the model will also have multiple levels of detail for more efficient visualization and analysis according to different user and application requirements. 


\section{POINT CLOUDS AND MODELS}

As described previously, 3D point clouds can be acquired directly using laser scanners or generated from multi-view images using photogrammetric algorithms. In recent years, structure from motion (SfM) and related algorithms (e.g., Faugeras and Lustman, 1988; Rothermel et al., 2012; Wu., 2011, 2013) have become an popular method of choice to generate 3D point clouds from multiple 2D images. In addition to freeware, commercial software such as Photoscan, ContextCapture (Acute3D) and Pix4D are also widely used to create 3D digital surface models of various sites and objects from ground-based or UAV (Unmanned Aerial Vehicles) images. This type of 3D modeling or reconstruction has also been successfully applied to cultural heritage applications (Dellepiane et al., 2013; Stathopoulou et al., 2015; Sun and Cao, 2015; Wenzel et al., 2013) for it does not require expensive equipments and has lower requirements on operations.

Whether the data are acquired using laser scanners or generated with image-based photogrammetric algorithms, and no matter how dense the data are, 3D point clouds are still discrete points. Although the points may have accurate 3D coordinates, there is no topographic relationship recorded in the original data sets. Therefore, the point clouds are subsequently processed and analyzed to produce 3D volumetric or surface models. Recently, after acquiring or generating the 3D point clouds of a cultural heritage site, building, or object, a subsequent processing is usually employed in order to create $3 \mathrm{D}$ models of the target. A conventional approach is to construct CAD (computer-aided design) style models in parametric or polyhedral formats. Another emerging strategy is to directly connect the 3D points to generate triangulated or grid-based mesh networks as a digital surface model (DSM) of the target. Both approaches have their advantages and disadvantages as summarized and listed in Table- 1 . For example, on the one hand, parametric or polyhedral models usually requires less storage space and can be easily constructed as object or element-based models to maintain the structural characteristics of the targets but they are difficult to show complex surfaces or small structures well. On the other hand, mesh-based models can describe complex surfaces in fine details, but they usually have larger data volumes to store all vertices and connections. In addition, although mesh-based models can describe fine details of complex surfaces, they are difficult to maintain the elements or structural property of the targets. In order to take advantages of both approaches, this study developed a framework to integrate 3D volume (parametric or polyhedral) and mesh models for representing complicated cultural heritage buildings in multiple levels of detail.

Table 1. Comparison of parametric/polyhedral and mesh-based modeling approaches from 3D point clouds

\begin{tabular}{|c|c|c|}
\hline Model Type & Advantages & Disadvantages \\
\hline $\begin{array}{l}\text { Parametric/ } \\
\text { Polyhedral }\end{array}$ & $\begin{array}{l}\text {-low storage } \\
\text {-object-based } \\
\text {-maintain structural } \\
\text { property }\end{array}$ & $\begin{array}{l}\text {-difficult to show } \\
\text { details }\end{array}$ \\
\hline Mesh & $\begin{array}{l}\text {-show complex } \\
\text { surface well }\end{array}$ & $\begin{array}{l}\text {-large data volume } \\
\text {-less structural } \\
\text { property }\end{array}$ \\
\hline
\end{tabular}

\section{PROPOSED METHODOLOGY}

The proposed 3D modeling framework is separated into three phases. The first stage is the processing of $3 \mathrm{D}$ point clouds. One of the most important task in this stage is the segmentation of point clouds. Effective point clouds segmentation algorithms are available for different data sources, applications and other considerations (Grilli et al., 2017). The objective of segmentation is to partition the data into different groups pertaining to different structure elements, which will correspond to different model components of the building.

The second phase is to generate a bare-bones volume model consisting of parametric, prismatic, or polyhedral components. The process involves identifying required parameters to shape segmented point clouds into parametric or polyhedral model components describing individual building elements (Teo and Chiu, 2015), and then connecting all components using CSG (Constructive Solid Geometry) or similar algorithms to form a barebones model representing the skeleton structure of the target. If necessary, additional levels of detail of the bare-bones model can be achieved using procedural (Koehl and Roussel, 2015) or projected generalization (Tsai et al., 2012) algorithms. Figure 1 is an example bare-bones volume model generated from a complicated point clouds of a Chinese style Land God (Tu Di Gong) shrine commonly seen in Chinese descendent communities across China and Southeast Asia. The bare-bones model not only represents the basic geometric shape and formation of the building; it also maintains the fundamental structural attribute of the building.

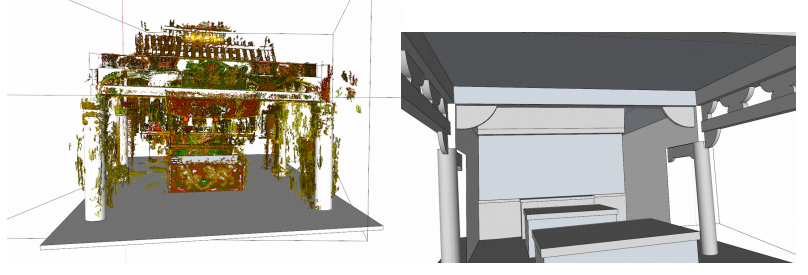

Figure 1. Point clouds of a Chinese style Land God shrine and its reconstructed bare-bones volume model.

The last phase is to add ornaments, decorations, figurines and other minor and surface structures onto the bare-bones model. Before doing so, the point clouds are filtered using the barebones model as a mask to exclude unnecessary points. The remaining points are connected using Poisson surface reconstruction or other algorithms to form a surface models of different parts. The final step is to connect the mesh objects onto the barebones model. Lai and Yuen (2015) demonstrated generating a transitional connection triangulated network between a mesh and a NURBS (non-uniform rational B-Spline) surface to seamlessly blend mesh objects onto parametric surfaces. Their transitional connection network is generated based on parametrized boundary conditions, control boundary points, and energy minimization constraints. These algorithms require intensive computation and may not be applicable to other types of parametric models. Therefore, a more general algorithm is developed to connect freeform meshes to parametric and polyhedral model surfaces. The procedure is straightforward: (1) first identify boundary points of the mesh by examining the connectivity of mesh vertices; (2) calculate the tangent vector of each boundary vertex; (3) project the boundary tangent vectors onto bare-bones model surface and find the intersections; (4) finally connect the boundary mesh vertices to the corresponding intersection points. This algorithm requires less computation but can still maintain $\mathrm{C}-1$ continuity between the mesh and bare-bones model connection. 


\section{EXPERIMENTAL RESULTS}

The proposed strategy for generating hybrid models of complicated cultural heritage buildings was first applied to an opensource 3D point cloud data set to test its validity and performance. The open-source 3D point cloud data set was generated by Seitz et al. (2006) for comparing and evaluating different milt-view stereo reconstruction algorithms and is available at $<$ http://vision.middlebury.edu/mview/data/ $>$. The point cloud was generated from 312 images sampled on a hemisphere of a plaster reproduction of "Temple of the Dioskouroi" in Agrigento, Sicily, Italy and thus is noted as the "temple test case" thereinafter. Figure- 2 shows an image of the plaster model and the preliminarily segmented point clouds. Figure- 3 shows two views of the reconstructed bare-bones model. As displayed in the figure, the reconstructed bare-bones model has effectively represent the skeleton of the building, which also provides an intuitive presentation of the components making up the entire buildings. As a result, in addition to the skeleton shape and geometric characteristics, the model also preserves the fundamental structural property of the building.
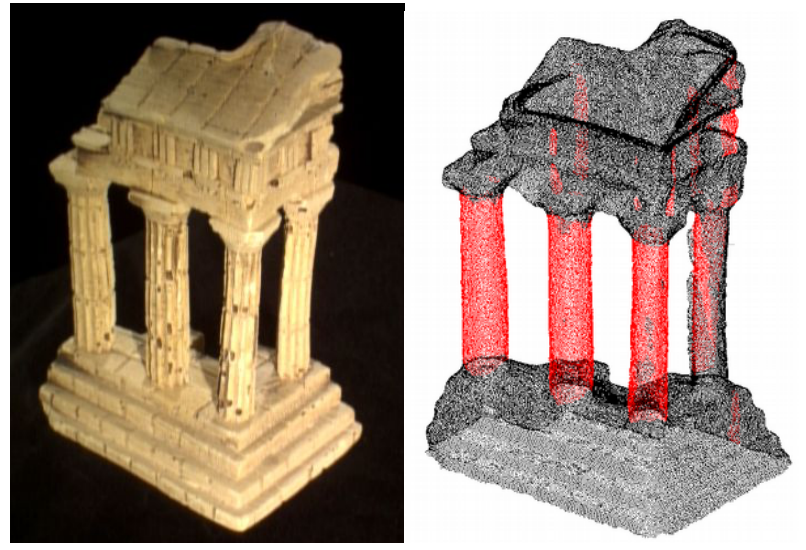

Figure 2. Temple of the Dioskouroi and preliminarily segmented $3 \mathrm{D}$ point clouds.

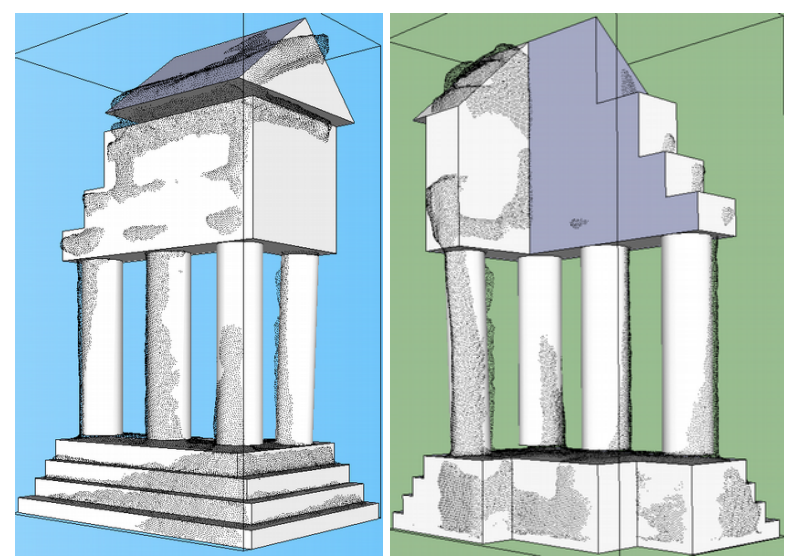

Figure 3. Reconstructed bare-bones model from the temple 3D point clouds. The original points are also shown in the figure.

Figure-4 displays the reconstructed full mesh model and a hybrid model of the temple test case. The full-mesh model utilized all original point clouds and consists of hundreds of thousand triangles. The hybrid model can significantly reduce the data amount but still provides fine details of complex surfaces; more important, it also preserves elemental structural characteristics, as provided by the skeleton bare-bones model, of the building.

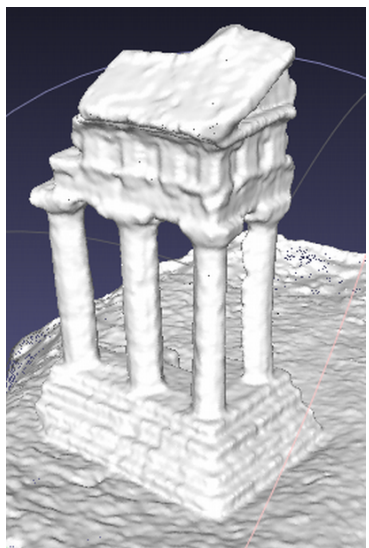

(a) full mesh model

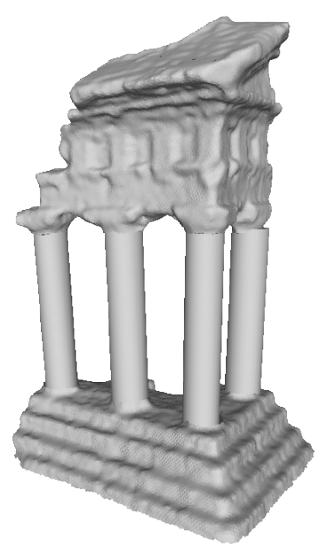

(b) hybrid model
Figure 4. Full-mesh and hybrid models of the temple test case.

The second test data set is the 3D point clouds of the North Gate of the old Taipei city wall. The construction of Taipei city wall was completed in 1884. However, the wall (except four of the five gates and guarding towers) was completely destroyed by the Japanese colonial government in 1905, 10 years after Japan occupied Taiwan. Among the four surviving gates, the North Gate (Cheng-En Gate) is the most well-preserved and the leastmodified one. Two photographs of this heritage structure taken in the 1950's and modern time are displayed in Figure 5. The 3D point clouds were generated from a 3D model reconstructed using single-view 3D metrology techniques (Chang and Tsai, 2012). Figure 6 illustrates the generated point clouds and two types of mesh-based surface models constructed from the point clouds. Examples of the reconstructed bare-bones and hybrid models are displayed in Figure 7. As can be seen in Figure 7, the hybrid model not only can preserve the basic structural attribute of the entire building; it can also describe the fine details of the roof tiles and ornaments.

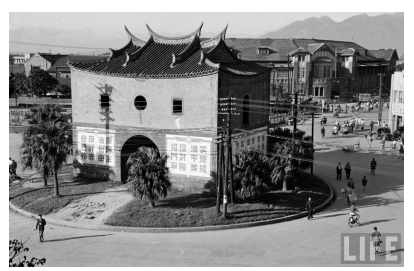

(a) in 1950's (c)Time Magazine

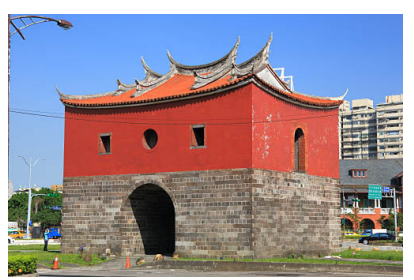

(b) in 2010's
Figure 5. Photographs of the Taipei City Wall North Gate taken in the 1950's and modern time.

\section{CONCLUSION}

This study developed a coarse-to-fine strategy that combines parametric or polyhedral type models and mesh objects for representing complicated cultural heritage buildings. The developed modeling framework is separated into three phases, including segmentation, bare-bones skeleton model generation, and integration of mesh objects. Although still a work in progress, example results of two test data sets demonstrated in this paper indicate that 


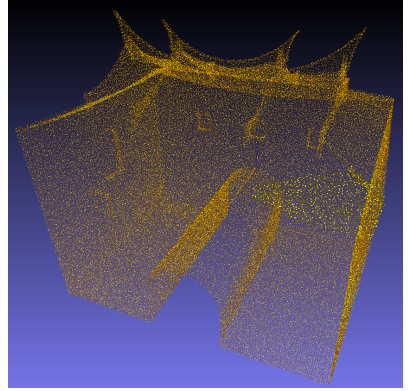

(a) $3 \mathrm{D}$ point clouds

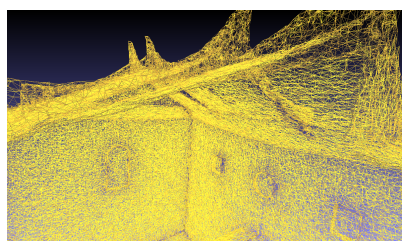

(b) irregular mesh surface model

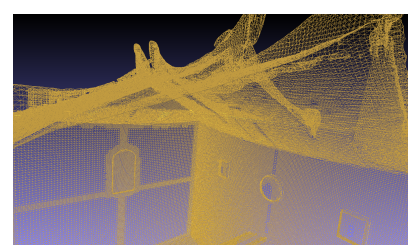

(c) regular mesh surface model
Figure 6. Point clouds of the North Gate test case and reconstructed mesh-based surface models.
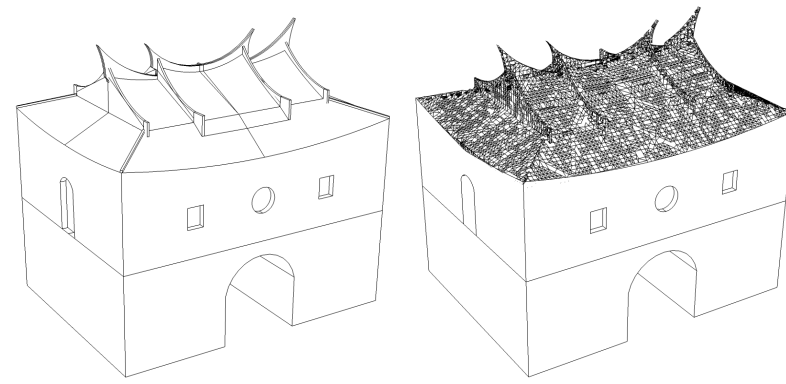

Figure 7. Polyhedral (left) and hybrid (right) models of the Taipei North Gate providing different levels of detail.

using the proposed hybrid modeling approach can significantly reduce the necessary data points to retain the fundamental volume and structural attributes and to preserve detailed elements and surface appearances of complicated heritage buildings. The proposed approach also gives the reconstructed 3D models multiple levels of detail to fulfill different user and application requirements. However, a reasonable and systematic LOD definition criteria should be established for this process to be carried out more smoothly. Future improvement of the proposed hybrid modeling framework will also include increase the robustness of mesh and parametric surface blending and increase the degree of automation.

\section{ACKNOWLEDGEMENTS}

This research was supported, in part, by the Ministry of Interior of Taiwan (ROC) under Project No. SYC1060303. The authors would also like to thank Prof. Tee-Ann Teo at the National Chiao Tung University of Taiwan for his valuable suggestions about 3D point cloud processing and modeling algorithms.

\section{References}

Alshawabkeh, Y., Balawi, F. and Haala, N., 2010. 3D digital documentation, assessment, and damage quantification of the Al-
Deir monument in the ancient city of Petra, Jordan. Conservation and Management of Archaeological Sites 12(2), pp. 124 145 .

Chang, H. and Tsai, F., 2012. Reconstructing three-dimensional specific curve building models from a single perspective view image. International Archives of the Photogrammetry, Remote Sensing and Spatial Information Sciences XXXIX-B6, pp. 101-106.

Dellepiane, M., Dell'Unto, N., Callieri, M., Lindgren, S. and Scopigno, R., 2013. Archaeological excavation monitoring using dense stereo matching techniques. Journal of Cultural Heritage 14(3), pp. 201.

Faugeras, O. D. and Lustman, F., 1988. Motion and structure from motion in a planar environment. International Journal of Pattern Recognition and Artificial Intelligence 2(3), pp. 485508.

Grilli, E., Menna, F. and Remondino, F., 2017. A review of point clouds segmentation and classification algorithms. International Archives of the Photogrammetry, Remote Sensing and Spatial Information Sciences XLII-2/W3, pp. 339-344.

Jamhawi, M., Alshawabkeh, Y., Freewan, A. and Al-Gharaibeh, R., 2016. Combined laser scanner and dense stereo matching techniques for $3 \mathrm{~d}$ modelling of heritage sites: Dar esSaraya museum. Mediterranean Archaeology and Archaeometry 16(3), pp. 185-192.

Koehl, M. and Roussel, F., 2015. Procedural modelling for reconstruction of historic monuments. ISPRS Annals of the Photogrammetry, Remote Sensing and Spatial Information Sciences II-5/W3, pp. 137-144.

Koller, D., Frischer, B. and Humphreys, G., 2009. Research challenges for digital archives of 3D cultural heritage models. ACM Journal on Computing and Cultural Heritage 2(3), pp. 7:1$7: 16$.

Koutsoudis, A., Vidmar, B., Ioannakis, G., Arnaoutoglou, F., Pavlidis, G. and Chamzas, C., 2014. Multi-image 3D reconstruction data evaluation. Journal of Cultural Heritage 15(1), pp. 73-79.

Lai, L. M. and Yuen, M. M. F., 2015. Blending of mesh objects to parametric surface. Computers \& Graphics 46, pp. 283-293.

Münster, S., Kuroczyński, P., Pfarr-Harfst, M., Grellert, M. and Lengyel, D., 2015. Future research challenges for a computerbased interpretative 3D reconstruction of cultural heritage - a German community's view. ISPRS Annals of the Photogrammetry, Remote Sensing and Spatial Information Sciences II5/W3, pp. 207-213.

Rothermel, M., Wenzel, K., Fritsch, D. and Haala, N., 2012. SURE: Photogrammetric surface reconstruction from imagery. In: Proceedings LC3D Workshop, Berlin, Germany.

Seitz, S., Curless, B., Diebel, J., Scharstein, D. and Szeliski, R., 2006. A comparison and evaluation of multi-view stereo reconstruction algorithms. In: CVPR2006, Vol. 1, pp. 519-526.

Stathopoulou, E. K., Georgopoulos, A., Panagiotopoulos, G. and Kaliampakos, D., 2015. Crowdsourcing lost cultural heritage. ISPRS Annals of the Photogrammetry, Remote Sensing and Spatial Information Sciences II-5/W3, pp. 295-300. 
Sun, Z. and Cao, Y. K., 2015. Data processing workflows from low-cost digital survey to various applications: Three case studys of Chinese historic architecture. International Archives of the Photogrammetry, Remote Sensing and Spatial Information Sciences Volume XL-5/W7, pp. 409-416.

Teo, T.-A. and Chiu, C.-M., 2015. Pole-like road object detection from mobile lidar system using a coarse-to-fine approach. IEEE Journal of Selected Topics in Applied Earth Observations and Remote Sensing 8(10), pp. 4805-4818.

Tsai, F., Lin, W.-J. and Chen, L.-C., 2012. Multi-resolution representation of digital terrain and building models. In: W. Shi, M. Goodchild, B. Lees and Y. Leung (eds), Advances in Geo-
Spatial Information Science, Vol. 10, ISPRS Book Series, pp. 233-242.

Wenzel, K., Rothermel, M., Fritsch, D. and Haala, N., 2013. Image acquistion and model selection fro multi-view stereo. International Archives of the Photogrammetry, Remote Sensing and Spatial Information Sciences XL-5/W1, pp. 251-258.

Wu., C., 2011. VisualSFM: A visual structure from motion system. In: http://ccwu.me/vsfm/.

Wu., C., 2013. Towards linear-time incremental structure from motion. In: $3 D V$. 